Prof. Dr. med. M. Nauck

Diabeteszentrum Bad Lauterberg

Tagungspräsident der 45. Jahrestagung

der Deutschen Diabetes-Gesellschaft

in Stuttgart
37_ Diabetes in der

Schwangerschaft

42- Typ-2-Diabetes bei Kindern

\title{
Diabetestherapie in Bewegung
}

\section{Der kardiovaskulären Katastrophe vorbeugen - von Anfang an!}

— Die Zahl übergewichtiger Menschen steigt seit den 1970er-Jahren stetig und hat mittlerweile alarmierende Ausmaße angenommen. Da Übergewicht ein wesentlicher Risikofaktor für das Entstehen eines Typ-2-Diabetes ist, folgt der Welle der Adipositas mit wenigen Jahren Verzögerung unweigerlich eine Diabetesepidemie. Die starke Ausbreitung von Adipositas und Diabetes ist wohl auf grundlegende Lebensstiländerungen zurückführen, die sich mit den Stichworten ungesunde (hyperkalorische) Ernährung und Bewegungsmangel nur unzureichend charakterisieren lassen. Am Ende dieser Entwicklung stehen u.a. gehäuft kardiovaskuläre Katastrophen wie Herzinfarkt, Schlaganfall und andere Folgen von Gefäßverschlüssen.

\section{Prävention beginnt bei den Kleinsten}

Die Basis dieser Gesundheitsprobleme wird geschaffen, lange bevor der Mensch einen Arzt aufsucht, um seine Krankheit behandeln zu lassen. Wenn also Vorbeugung unser Ziel ist, kann dies, mithilfe fachlicher medizinischer Ratschläge, nur dort stattfinden, wo die betroffenen Menschen abgeholt werden können. Aktuellen Erkenntnissen zufolge sind solche Interventionen umso erfolgreicher, je früher im Leben sie stattfinden. Hier sind also Programme gefragt, die sich für Kindergärten und Schulen eignen bzw. für eine Begleitung von Menschen im Beruf. Die Zauberformel ist ein mittelintensives Training der Ausdauerfitness, nicht durch extreme sportliche Belastungen, sondern indem man den Alltagsherausforderungen (Treppen steigen statt Aufzug fahren, Bewegung zu Fuß und auf dem Fahrrad) nicht aus dem Weg geht. Vielmehr soll man diese mindestens fünfmal pro Woche 30 Minuten lang ausdrücklich suchen. Deshalb hat die Deutsche Diabetes-Gesellschaft für den diesjährigen 45. Jahreskongress das Motto "Diabetestherapie in Bewegung“ gewählt. Ist ein Diabetes erst manifest und sind möglicherweise die frühen, nach einfachen Therapieschemata zu behandeInden Stadien vorbei, müssen antidiabetische Medikamente eingesetzt werden.

Die Größe des ungelösten Problems bringt es mit sich, dass derzeit zahlreiche neue Wirkstoffklassen entwickelt werden. Die neuen
Medikamente sehen häufig auf den ersten Blick deutlich „sympathischer" aus, weil sie z.B. die Nachteile herkömmlicher Wirkstoffe wie Gewichtszunahme und Hypoglykämiegefahr nicht mitbringen. Der Nachweis, dass neue Medikamente wie die GLP-1-RezeptorAgonisten (Inkretin-Mimetika) und Hemmstoffe des Enzyms Dipeptidyl-Peptidase-4 (DPP-4-Hemmer) den Blutzucker und $\mathrm{HbA}_{1 \mathrm{c}}$ deutlich senken, ohne Hypoglykämien auszulösen, und dass entweder gar kein Einfluss auf das Körpergewicht ausgeübt wird (DPP4-Hemmer) oder sogar mit einer soliden Gewichtsabnahme zu rechnen ist (Inkretin-Mimetika), ersetzt natürlich nicht den Nachweis, dass diese Medikamente Folgeerkrankungen eines Diabetes verhindern können. Doch solange Studien mit solchen Endpunkten noch laufen (was glücklicherweise der Fall ist), kann man sich naturgemäß noch nicht auf ihre Ergebnisse berufen. Deshalb wird in einem kostenbewussten Umfeld mehr oder weniger Druck ausgeübt, diese neuen Medikamente nur bei sehr guter individueller Prüfung einzusetzen oder ihren Einsatz von vornherein zu begrenzen.

\section{Langzeitstudien sind teuer, aber notwendig}

An dieser Stelle sind einige mahnende Worte zur künftigen Entwicklung in unserem Gesundheitssystem angebracht: Der Nachweis einer verminderten Häufigkeit von Folgeerkrankungen durch eine blutzuckersenkende Behandlung dauert nach neuen Erkenntnissen sehr lang. Angemessen erscheinen mindestens 8-15 Jahre. Dass dies mit vergleichsweise hohen, um nicht zu sagen übermäßigen Kosten verbunden ist, ist eine große Gefahr. Wegen des hohen Aufwands könnten Ressourcen in die Untersuchung von Therapien akuter Erkrankungen umgeleitet werden, deren Erforschung weniger Aufwand und Zeit kostet. Dies würde Menschen mit Typ-2-Diabetes im engeren und chronisch Kranke im weiteren Sinn langfristig stark benachteiligen und ihnen therapeutische Chancen nehmen, die ihnen eigentlich zustehen. All diejenigen, die an der Gestaltung von Versorgungsstrukturen und politischen Rahmenbedingungen mitwirken, sollten dies in ihre Überlegungen einbeziehen. 